University of Wollongong

Research Online

Faculty of Engineering - Papers (Archive)

Faculty of Engineering and Information

Sciences

2005

\title{
Third harmonics due to surface barrier in high-temperature superconductor
}

X. B. Xu

Nanjing University, China

L. Zhang

Nanjing University, China

X. Leng

Nanjing University, China

Shichao Ding

University of Wollongong, sding@uow.edu.au

Hua-Kun Liu

University of Wollongong, hua@uow.edu.au

See next page for additional authors

Follow this and additional works at: https://ro.uow.edu.au/engpapers

Part of the Engineering Commons

https://ro.uow.edu.au/engpapers/168

\section{Recommended Citation}

Xu, X. B.; Zhang, L.; Leng, X.; Ding, Shichao; Liu, Hua-Kun; Wang, Xiaolin; Dou, S. X.; Lin, Z. W.; and Zhu, J. G.: Third harmonics due to surface barrier in high-temperature superconductor 2005.

https://ro.uow.edu.au/engpapers/168

Research Online is the open access institutional repository for the University of Wollongong. For further information contact the UOW Library: research-pubs@uow.edu.au 
Authors

X. B. Xu, L. Zhang, X. Leng, Shichao Ding, Hua-Kun Liu, Xiaolin Wang, S. X. Dou, Z. W. Lin, and J. G. Zhu 


\title{
Third harmonics due to surface barrier in high-temperature superconductor
}

\author{
X. B. Xu, L. Zhang, X. Leng, and S. Y. Ding ${ }^{\text {a) }}$ \\ National Laboratory of Solid State Microstructures, Department of Physics, Nanjing University, \\ Nanjing 210093, People's Republic of China
}

H. K. Liu, X. L. Wang, and S. X. Dou

Institute for Superconducting and Electronic Materials, University of Wollongong, New South Wales 2522, Australia

\author{
Z. W. Lin and J. G. Zhu \\ University of Technology Sydney, New South Wales 2007, Australia
}

(Presented on 8 November 2004; published online 2 May 2005)

\begin{abstract}
The influence of surface barrier on the third harmonics ac susceptibility $\left(\chi_{3}\right)$ is studied numerically. The surface barrier is described by a critical current density in surfaces which are higher than the inside one. The model can act as the critical state one, the flux creep one, or the flux flow one when temperature (or field) changes. The numerical results based on the model are more close to the popular experimental data probing the harmonics as a function of temperature (or field). Besides, the surface barrier will lead to new peaks in the real and imaginary parts of the third harmonics, which are the finger signature of the surface barrier. Comparison of the peaks with those of the real part of elemental harmonic shows that they are located at the same temperature where dips in $\chi^{\prime}$ caused by the surface barrier occur, which is in well agreement with experiments. (C) 2005 American Institute of Physics. [DOI: 10.1063/1.1851413]
\end{abstract}

\section{INTRODUCTION}

Surface barrier is important in understanding irreversible phenomena of superconductors. ${ }^{1-8}$ Experiments show that there exists a surface zone with a higher critical current density $j_{c}$ (larger than the inside one) which may originate from the surface pinning, the Bean-Livingston barrier, or the geometrical barrier. ${ }^{9-15}$ We have proposed a model to describe the surface barrier (SB) and studied the influence of SB on the fundamental ac susceptibility (ACS). ${ }^{16}$ In this paper, we will study SB's effects on the third harmonic ACS based on the same model. Usually ACS is measured as a function of temperature (or dc field). To understand the observations in experiments, different models have been proposed. ${ }^{4,17,18}$ However, it is still worthy to discuss if or not only one of these models can be employed for the whole of an ACS curve in all temperature regions, because the critical state model is effective at low temperatures, the flux creep model at the relatively higher temperatures, and the flux flow model in the regime near $T_{c}$. So we proposed a model of power-law flux velocity whose power $n$ is dependent on temperature (and field) [see below Eq. (1)]. The $n$ is large at low temperatures. With the increment of temperature, $n$ decreases and becomes zero at $T_{c}$. The model is in contrast with the previous ones (see, for example, Ref. 18 where $n \equiv \sigma$ ) whose power $n$ is independent of temperature (field). Our model may be more proper to explain the ACS data at different temperatures.

\footnotetext{
a) Author to whom correspondence should be addressed; electronic mail: syding@nju.edu.cn
}

\section{MODEL AND SIMULATION}

The essential point of our model is that there are two $j_{c}$ in a sample: the higher one $j_{\mathrm{cs}}$ in the surface zone and the lower one $j_{\mathrm{cb}}$ inside the bulk. We consider a one-dimensional case that the sample is infinite in the $y$ and $z$ directions, the field in the $z$ axis penetrates only along the $x$ axis, which is suitable not only for the Bean-Livingston barrier and the surface pinning, but also for the geometrical one of a long (in the $y$ axis) sample with rectangular cross section (in the $x$ $-z$ plane, thickness $t$ in the $z$ axis) in perpendicular field. ${ }^{9}$ The bulk width is $d_{b}$ whose dimension is millimeters for a bulk sample and micrometers for a thin film. We note that the surface width $d_{s}$ is comparable to the penetration depth $\lambda$ for the Bean-Livingston barrier, but $d_{s}$ is nearly half of the sample thickness $(t / 2)$ for the geometrical barrier. For the surface pinning barrier, it is also a relatively broad zone. It is clear that $d=2 d_{s}+d_{b}$. Therefore, we choose $d_{s} / d_{b}=0.1$ for different $d_{b}(1 \mu \mathrm{m}-1 \mathrm{~mm})$, and the strength of SB is adjusted by $j_{c 0 s} / j_{c 0 b}$ in the simulation. In fact, although $d_{s} / d_{b}$ is regulated from 0.1 to 0.001 for $j_{\mathrm{cs}} / j_{\mathrm{cb}}=10-100$, there is almost no difference in the numerical results.

The material equation is

$$
v=v_{0}\left(j / j_{c}\right)\left|j / j_{c}\right|^{n},
$$

where $v$ is the flux-line velocity, $v_{0}$ is a velocity scale, and $j$ is current density. From Eq. (1) the power law $E(j)$ $=E_{0}\left(j / j_{c}\right)^{n+1}$ is obtained, which results in the critical state model for $n \rightarrow \infty$, the flux creep one for finite $n$ and the flux flow one for $n=0$. The flux diffusion equation is 


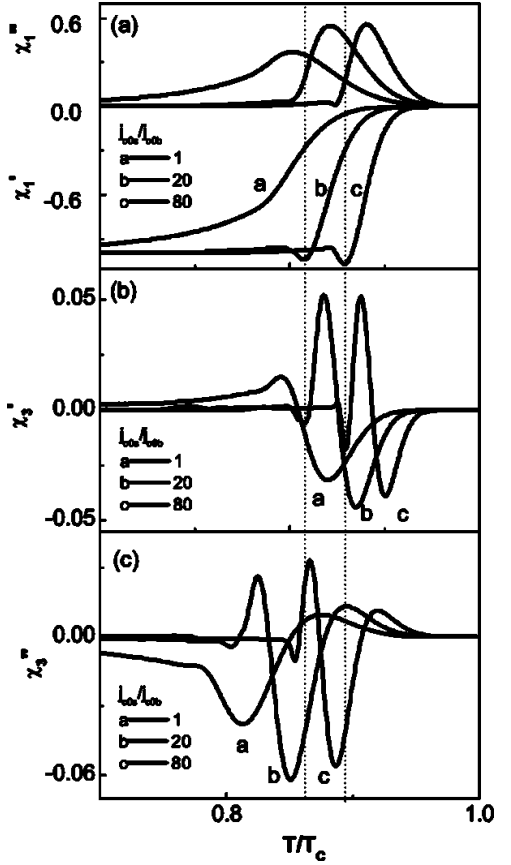

FIG. 1. The harmonics with $\left(j_{c 0 s} / j_{c 0 b}>1\right)$ and without $\left(j_{c 0 s} / j_{c 0 b}=1\right)$ the surface barrier as a function of temperature. The dotted lines show the dips originate from the surface barrier.

$$
\frac{\partial B}{\partial t}=\frac{v_{0}}{\left(\mu_{0} j_{c}\right)^{n+1}} \frac{\partial}{\partial x}\left[\left|\frac{\partial B}{\partial x}\right|^{n}\left(\frac{\partial B}{\partial x}\right)|B|\right] .
$$

The ac susceptibilities can be calculated by

$$
\chi_{n}=\chi_{n}^{\prime}-i \chi_{n}^{\prime \prime}=\frac{\mu_{0}}{\pi B_{\mathrm{ac}}} \int_{0}^{2 \pi} M(t) \exp (i n \omega t) d(n \omega t),
$$

where $M$ is magnetization. The temperature and field dependence of $j_{c}$ and $n$ are, respectively,

$$
\begin{aligned}
& j_{c}=j_{c 0}\left[1+\left(\frac{T}{T_{c}}\right)^{2}\right]^{-1 / 2}\left[1-\left(\frac{T}{T_{c}}\right)^{2}\right]^{5 / 2} \frac{B_{0}}{B_{0}+|B|}, \\
& n=n_{0} \frac{T_{c}}{T}\left[1-\left(\frac{T}{T_{c}}\right)^{4}\right] \frac{B_{0}}{B_{0}+|B|} .
\end{aligned}
$$

We note that this $n$ is large at low temperatures but small at high temperatures, which is very important to assure that our model works well in various temperature regimes. In the simulation we let $n_{0}=5, v_{0}=1 \mathrm{~m} / \mathrm{s},{ }^{19} j_{c 0 b}=5 \times 10^{8} \mathrm{~A} / \mathrm{m}^{2}$, and $B_{0}=500$ Gs.

For comparison, the critical state model is also calculated in this paper, where $j_{c}=j_{\text {cs }}$ or 0 at the surface and $j_{c}$ $=j_{\mathrm{cb}}$ or 0 in the bulk. For the field dependence, we also use the Kim model $j_{c}(B)=j_{c 0}\left[B_{0} /\left(B_{0}+|B|\right)\right]$.

With the finite difference method, the nonlinear diffusion equation can be numerically solved. And the implicit difference scheme is used for stability.

\section{RESULTS AND DISCUSSIONS}

The third $\left(\chi_{3}^{\prime}, \chi_{3}^{\prime \prime}\right)$ and the first $\left(\chi_{1}^{\prime}, \chi_{1}^{\prime \prime}\right)$ harmonics with SB $\left(j_{c 0 s} / j_{c 0 b}>1\right)$ and without SB $\left(j_{c 0 s} / j_{c 0 b}=1\right)$ are shown in Fig. 1. If there is no SB, two peaks (we call them primary

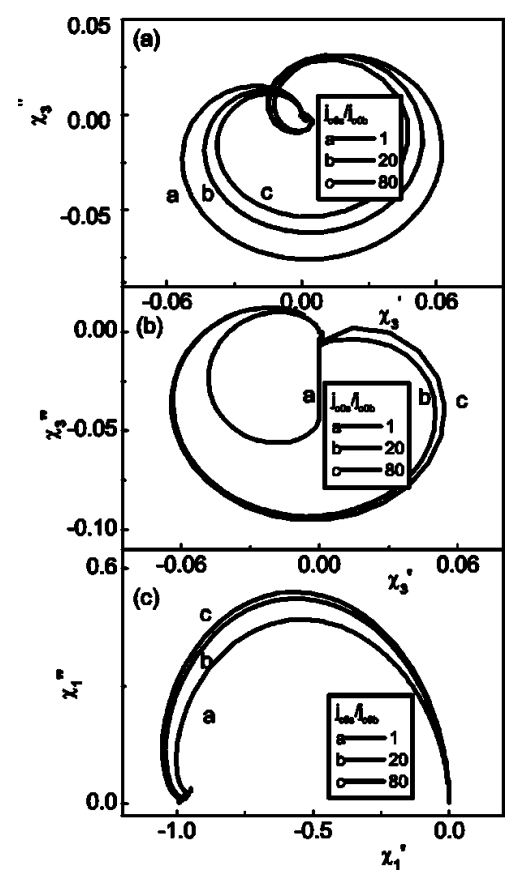

FIG. 2. Polar plots of the third and the first harmonics with $\left(j_{c 0 s} / j_{c 0 b}>1\right)$ and without $\left(j_{c 0 s} / j_{c 0 b}=1\right)$ the surface barrier. (a), (c) The harmonics with temperature power law $n$. (b) the third harmonics with constant $n$.

peaks) appear in both the $\chi_{3}^{\prime}\left[\right.$ Fig. $1(\mathrm{~b})$, curve a] and $\chi_{3}^{\prime \prime}[$ Fig. $1(\mathrm{c})$, curve a], respectively, which is similar to the previous results. ${ }^{20,21}$ Accordingly, $\chi_{1}^{\prime}$ and $\chi_{1}^{\prime \prime}[$ Fig. 1(a), curve a] have no dips. ${ }^{16}$ We can see that the new feature occurs when SB is considered. The most important one is that additional and smaller negative peaks come out in $\chi_{3}^{\prime}$ and $\chi_{3}^{\prime \prime}$, respectively. When SB becomes stronger, two or more small peaks appear. Besides, because SB can hold back the entrance of the flux lines and only at higher temperatures the flux lines can penetrate into the sample, the peaks shift to higher temperatures with the increasing barrier strength. On the other hand, the primary peaks are not monotonically increasing with $j_{c 0 s} / j_{c 0 b}$ though they are all in some measure larger than the ones without SB. When $j_{c 0 s} / j_{c 0 b}>20$, the heights of the two primary peaks reverse. This is similar to the sign reversal effect predicted in Ref. 17, where the third harmonics (both the real and the imaginary parts) change sign in transition from the geometrical barrier to the bulk pinning. What happened at the temperature where the additional peaks occur in the third harmonics? By comparing Fig. 1, one can easily see that the corresponding dips in $\chi_{1}^{\prime}$ just take place at the same temperature where the $\chi_{3}^{\prime}$ peaks occur, as indicated by the dotted lines. The dips in $\chi_{1}^{\prime}$ indeed have been observed by ACS and Hall array measurements. ${ }^{12,16}$ Therefore, we conclude that the additional and smaller negative peaks arise from SB.

As mentioned above, the velocity $v$ of the flux lines (the material equation) changes with temperature and field because $n$ is dependent on $T$ and $B$. For the ACS curves in Fig. 1 , at low temperatures where $v$ is very small, the critical state model $(v=0)$ is proper, where increasing temperature flux lines move more and more faster, flux creep model goes into effect, and near $T_{c}$ the flux lines will flow and the linear material equation should be taken into account. Therefore the 
ACS curves are, in fact, calculated by the critical state, the flux creep, and the flux flow models as temperature goes up. This is very different from the reported results, where either the critical state model or the flux creep one was used for the whole of a $\chi(T)$ curve. $^{18,22}$

Shown in Fig. 2 are the polar plots $\chi_{1}^{\prime \prime}-\chi_{1}^{\prime}$ and $\chi_{3}^{\prime \prime}-\chi_{3}^{\prime}$. It is seen that the results in Figs. 2(a) and 2(c) are also different from the reports in Refs. 4 and 18. The cardiodids which traverse in clockwise sense upon decreasing temperature are distorted. In fact, the distortion results from SB and the alteration of the flux creep velocity due to temperature. To confirm this understanding, we calculated the plots $\chi_{3}^{\prime \prime}-\chi_{3}^{\prime}$ in terms of the so-called whole critical state model ( $n$ is infinite) which is suitable at any temperatures, including $T \approx T_{c}$ where $n=0$ [see Fig. 2(b)]. It is noted that these cardiodids are quite similar to the reports. 4,18

\section{SUMMARY}

In summary, we have used a model to study the influence of SB on the third and the first harmonics of ACS. The model can act as the critical state one, the flux creep one, or the flux flow one when temperature (or field) changes, which is more close to the usual situation in experiments. Simulation shows that SB has its own characteristic peaks (or dips) in the third harmonics, which can be considered as a finger signature of $\mathrm{SB}$. By comparing the peaks with those in the real part of elemental harmonic, we find that the peaks are at the same temperature where dips in $\chi^{\prime}$ caused by the SB occur, which coincides with experiments.

\section{ACKNOWLEDGMENTS}

The Ministry of Science and Technology of China (NKBRSFG1999-0646) and NNSFC (No. 19994016) support the work. One of the authors (X.L.) is supported by Motorola subject prize.

${ }^{1}$ J. R. Clem, J. Appl. Phys. 50, 3518 (1979).

${ }^{2}$ M. Benkraouda and J. R. Clem, Phys. Rev. B 53, 5716 (1996).

${ }^{3}$ L. Burlachkov, Phys. Rev. B 47, 8056 (1993).

${ }^{4}$ C. J. van der Beek, M. V. Indenbom, G. D. Anna, and W. Benoit, Physica C 258, 105 (1996).

${ }^{5}$ D. T. Fuchs, E. Zeldov, T. Tamegai, S. Ooi, M. Rappaport, and H. Shtrikman, Phys. Rev. Lett. 80, 4971 (1998).

${ }^{6}$ Y. Yeshurun, A. P. Malozemoff, and A. Shaulov, Rev. Mod. Phys. 68, 911 (1996).

${ }^{7}$ S. Tochihara, H. Yasuoka, and H. Mazaki, Physica C 268, 241 (1996).

${ }^{8}$ Y. Kimishima, K. Kamimura, and Y. Ichiyanagi, Physica C 329, 17 (2000).

${ }^{9}$ E. Zeldov, A. I. Larkin, V. B. Geshkenbein, M. Konczykowski, D. Majer, B. Khaykovich, V. M. Vinokur, and H. Shtrikman, Phys. Rev. Lett. 73, 1428 (1994)

${ }^{10}$ D. T. Fuchs, et al., Nature (London) 391, 373 (1998).

${ }^{11}$ Y. Paltiel et al., Nature (London) 403, 398 (2000).

${ }^{12}$ Y. Paltiel et al., Phys. Rev. B 58, 014763 (1998).

${ }^{13}$ L. Burlachkov et al., Phys. Rev. B 50, 16770 (1994).

${ }^{14}$ C. J. van der Beek et al., Phys. Rev. Lett. 84, 4196 (2000).

${ }^{15}$ C. P. Bean and J. D. Livingston, Phys. Rev. Lett. 12, 14 (1964).

${ }^{16}$ X. Leng et al., Phys. Rev. B 68, 214511 (2003).

${ }^{17}$ G. M. Maksimova, D. Yu. Vodolazov, and I. L. Maksimov, Physica C 356, 67 (2001).

${ }^{18}$ J. Gilchrist and T. Dombre, Phys. Rev. B 49, 1466 (1996).

${ }^{19}$ V. M. Vinokur, M. V. Feigel'man, and V. B. Geshkenbein, Phys. Rev. Lett. 67, 915 (1991).

${ }^{20}$ P. Fabbricatore, S. Farinon, G. Gemme, R. Musenich, R. Parodi, and B. Zhang, Phys. Rev. B 50, 3189 (1994).

${ }^{21}$ M. J. Qin and C. K. Ong, Physica C 319, 41 (1999).

${ }^{22}$ L. Ji, R. H. Sohn, G. C. Spalding, C. J. Lobb, and M. Tinkham, Phys. Rev. B 40, 10936 (1989). 